\title{
Distributed CTL Model-Checking and counterexample search
}

\author{
M.C. Boukala \\ LSI, Computer Science department, USTHB \\ BP 32 El-Alia \\ Algiers, ALGERIA \\ boukala@lsi-usthb.dz \\ L. Petrucci \\ LIPN, CNRS UMR 7030, Université Paris XIII \\ 99, avenue Jean-Baptiste Clément \\ F-93430 Villetaneuse, FRANCE \\ Laure.Petrucci@lipn.univ-paris13.fr
}

Keywords: Petri nets, verification, distributed algorithms, temporal logic

\begin{abstract}
In this paper, we propose a distributed algorithm for CTL model-checking and a counterexample search whenever the CTL formula is not satisfied. The distributed approach is used in order to cope with the state space explosion problem. A cluster of workstations performs collaborative verification over a partitioned state space. Thus, every process involved in the distributed verification executes a labelling procedure on its own partial state space, and uses the parse tree of the CTL formula to evaluate sub-formulas and delay the synchronisations so as to minimise idle time. A counterexample search consists in a distributed construction of the tree-like corresponding to the failure executions. Some experiments have been carried out to evaluate the efficiency of this approach.
\end{abstract}

\section{INTRODUCTION}

The design of critical systems requires checking properties to ensure a high level of reliability. Formal specification and verification is a means towards that goal. Model-checking is a successful verification method based on reachability analysis [McM98, $\left.\mathrm{BBF}^{+} 01, \mathrm{BCM}^{+} 92, \mathrm{Hol03}, \mathrm{CES} 86\right]$. However, it often encounters the state space explosion problem when dealing with large and complex systems.

Different methods were designed to cope with this problem using compact representations and symbolic model-checking [Bry92], symmetries $\left[\mathrm{BBF}^{+} 01\right]$, on-the-fly verification $\left[\mathrm{BBF}^{+} 01\right]$, etc. Another approach consists in taking advantage of a distributed environment. The idea is to increase the computational power and more specifically a large available memory, by using a cluster of computers. The use of networks of computers can provide the resources required to achieve verification of industrial case studies.

The important feature of distributed algorithms is to solve a given problem by distributing the data among the participating stations with a small amount of coordination. One of the main issues in distributing model-checking algorithms is the partitioning of the state space among the individual computers. Distributed algorithms for state space generation for high level models, such as Petri nets, using clusters or parallel computers have been recently proposed in e.g. [KM04, GMS01, CGN98].

In the same context, several works proposed to distribute the model-checking of linear temporal logic (LTL) formulae [LS99, BBC03a, BvH03, BvMv04, BBC03b], with a distributed search of accepting cycles in Büchi automata. Distributed and parallel model checking of CTL logic 
[BCY02, BH05, Bou05], and modal mu-calculus [BBW02] were also studied. In [BP07] we presented a distributed verification of reachability, liveness and home state properties.

Here, we focus on enumerative distributed model-checking for CTL logic, performed in a backward manner as in [BH05]. We propose a distributed search of a counterexample whenever the formula is not satisfied, based on the construction of the tree-like structure [CJLV02] corresponding to the sub-graph where the negation of the formula holds. The paradigm used for our algorithms is distributed computing based on message passing on a MIMD architecture with a distributed memory.

The paper is organized as follows. In section 2, we shortly recall CTL basic definitions. Then, section 3 describes the distributed setting we use for the state space. Section 4 presents the approach for CTL model-checking in this distributed framework. The counterexample search in a distributed environment is presented in section 5. An implementation of this algorithm led to experimental results discussed in section 6 .

\section{THE COMPUTATION TREE LOGIC CTL}

CTL is a branching time logic [CES86], based on models where at each moment there may be several different possible futures. The syntax of CTL formulas is given in definition 1, where the most elementary expressions are atomic propositions. The set of atomic propositions is denoted by $A P$ with elements $p, q, r \ldots$.

Definition 1 (CTL syntax) The set of CTL formulae is inductively defined as follows:

- $p \in A P$ is a CTL formula.

- If $\varphi$ and $\psi$ are CTL formulae, then : $\neg \varphi, \varphi \wedge \psi$ and $\varphi \vee \psi$ are CTL formulae.

- If $\varphi$ and $\psi$ are CTL formulae, then: $E X \psi, E(\psi U \varphi)$ and $A(\psi U \varphi)$ are CTL formulae.

$E$ and $A$ are respectively the existential and universal path operators, expressing that a property is valid for some path and for all paths. The temporal operators $X$ and $U$ are called respectively next and until operators, expressing that a property is valid in the next state, and that $\psi$ is valid until $\varphi$ becomes valid. They must be in the scope of an existential or universal path operator.

The interpretation of the CTL formulae is defined over a Kripke structure:

Definition 2 (Kripke structure) $A$ Kripke structure is a 4-tuple $M=\left(S, T, \lambda, s_{0}\right)$ where:

- $S$ is a non-empty set of states,

- $T \subseteq S \times S$ associates with each state $s \in S$ its possible successors,

- $\lambda: S \longrightarrow 2^{A P}$, associates with each state $s \in S$ the set of atomic propositions $\lambda(s)$ valid in $s$.

- $s_{0} \in S$ is the initial state.

Definition 3 (Paths) $A$ path is an infinite sequence of states $\sigma=s_{0} s_{1} s_{2} \cdots \in S^{\omega}$ such that $\forall i \geq 0,\left(s_{i}, s_{i+1}\right) \in T$. The set of paths is denoted $\mathcal{P}$.

\section{Notations:}

- The (i+1)th element of $\sigma \in \mathcal{P}$ is denoted $\sigma[i]$.

- The set of paths starting in state $s \in S$ is $P_{M}(s)=\{\sigma \in \mathcal{P} \mid \sigma[0]=s\}$.

The semantics of CTL formulas is then defined as follows:

Definition 4 (CTL semantics) Let $p \in A P$ be an atomic proposition, $M=\left(S, T, \lambda, s_{0}\right)$ a Kripke structure, $s \in S$, and $\varphi, \psi C T L$ formulae. The satisfaction relation $\models$ is inductively defined by:

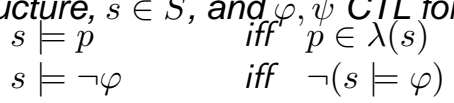




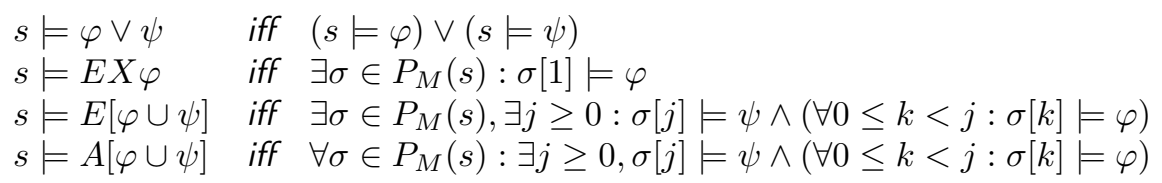

The interpretations for atomic propositions, negation and conjunction are as usual. $E X \varphi$ is valid in state $s$ if and only if there exists some path $\sigma$ starting in $s$ such that property $\varphi$ holds in the next state of this path, $\sigma[1]$.

$A[\varphi \cup \psi]$ is valid in state $s$ if and only if every path starting in $s$ has an initial finite prefix such that $\psi$ holds in the last state of this prefix and $\varphi$ holds at all other states along the prefix.

$E[\varphi \cup \psi]$ is valid in $s$ if and only if there exists a path starting in $s$ that satisfies the property $\varphi \cup \psi$.

It is said that $M \models \psi$ iff $s_{0} \models \psi$.

Some operators are also often defined and used in the literature: $E F, A F, E G, A G$ and $A X$, but they can all be obtained from those in definition 4 .

A model-checking algorithm for CTL consists in computing, for a given formula $\varphi$ and a model $M$, the subset: $\operatorname{Sat}(\varphi)=\{s \in S \mid s \models \varphi\}$ in an iterative manner. The problem of checking if $s \models \varphi$ is then reduced to checking if $s \in \operatorname{Sat}(\varphi)$. Sat is constructed inductively using the formula parse tree (see e.g. [CES86, IB06]).

\section{DISTRIBUTED STATE SPACE EXPLORATION}

Among the approaches to render verification amenable, distributed state space exploration consists in splitting the state space on different machines so as to take advantage of their memory. The partitioning of the state space is a crucial issue. Compact state representations, load balancing policies [KM04, LS99, GMS01], and similar approaches [CCBF94, CGN98] yield very good speedups.

The idea underlying a distributed algorithm for state-space generation is to use multiple processes to perform the exploration concurrently on distinct parts of the state space. Let us assume there are $\mathrm{N}$ machines connected by a network and communicating by message passing. To distribute the states among the different machines, we use a hash function $h: S \longrightarrow\{0, \ldots, N-1\}$. Then $h(s)$ is the owner of state $s$, i.e. the process responsible for storing $s$ and exploring its successors. This defines a partition of $S$ into $N$ sets: $S_{n}=\{s \mid h(s)=n\}$ for $n \in\{0, \ldots, N-1\}$.

The hash function must give a uniform partitioning and minimum cross arcs. A cross arc is an arc from a state $s$ to a state $s^{\prime}$ such that $h(s) \neq h\left(s^{\prime}\right)$, i.e. $s$ and $s^{\prime}$ do not belong to the same station. A large number of cross arcs will necessarily lead to more communications. Hence the choice of the hash function is a crucial issue for the technique to be efficient. In general, it could either be supplied by the user or be automatically computed. The computation could be similar to symmetries computations or be derived from an initial partial state space exploration.

As in [KP04], we use two types of processes: A coordinator and $N$ worker processes. The coordinator, which is a very light process, can be executed on the same station as any worker process. It initiates the generation, by sending the initial state $s_{0}$ to the worker process $h\left(s_{0}\right)$, and detects termination of the overall computation. Workers execute the same code ; a worker process $i$ is executed by station $i$, to generate the subset of states $S_{i}$ owned by this station.

In the following, for generation as well as for verification, we describe only the algorithms executed by the worker processes. The generation algorithm executed by a machine $i$ (worker ${ }_{i}$ ) is briefly described in algorithm 1 , 


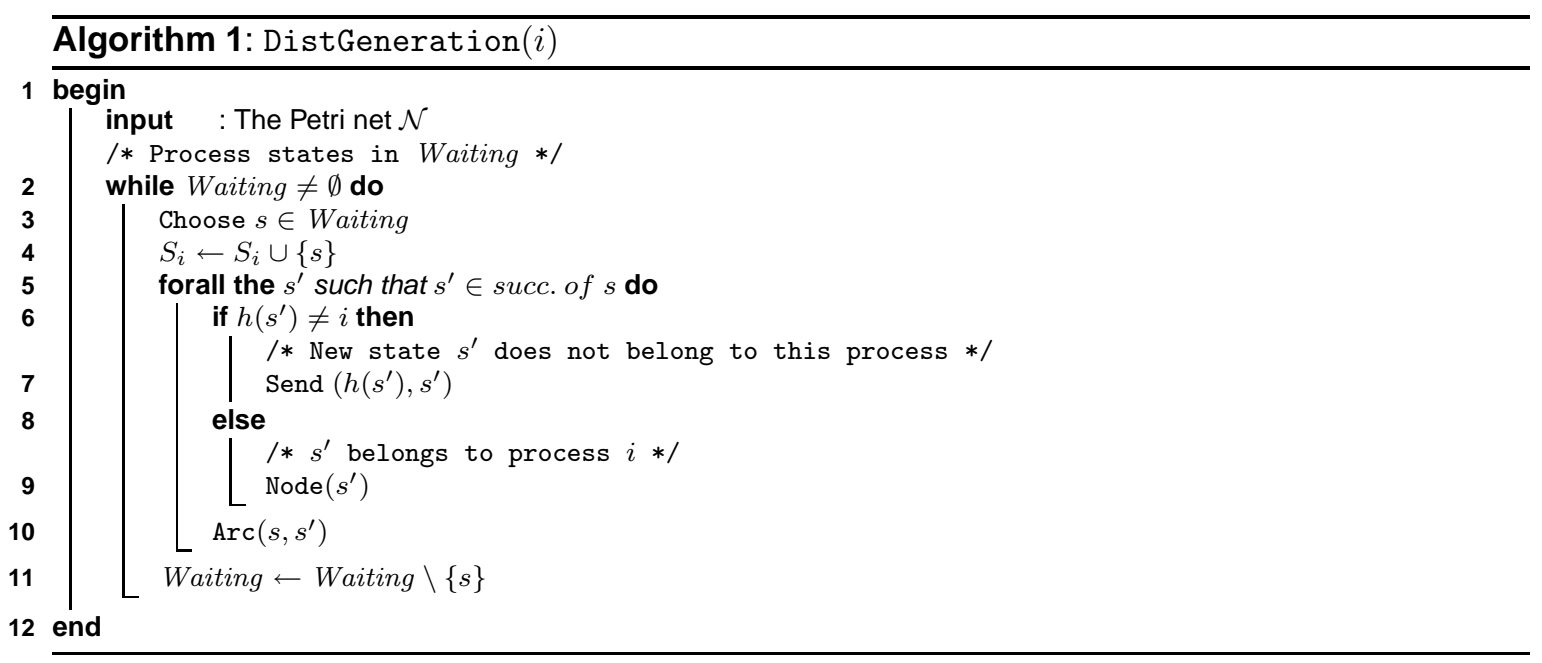

In the algorithm, Waiting contains the states which are not yet explored. At each iteration an element $s$ of Waiting is removed to generate its successors. Node $\left(s^{\prime}\right)$ adds local successors to Waiting if they have not been explored yet, distant successors are sent to their owner process. $\operatorname{Arc}\left(s, s^{\prime}\right)$ adds the arc $\left(s, s^{\prime}\right)$ to the set of arcs. Arcs can be stored using either a successors or a predecessors list, and every successor is marked by its owner process identification, in particular for distant successors.

The communications are asynchronous, thus, when receiving a message, the processes are preempted and a handler function MessHandler () is executed. The excerpt of this function shown in algorithm 2, indicates the operations executed when a state is received by station $i$.

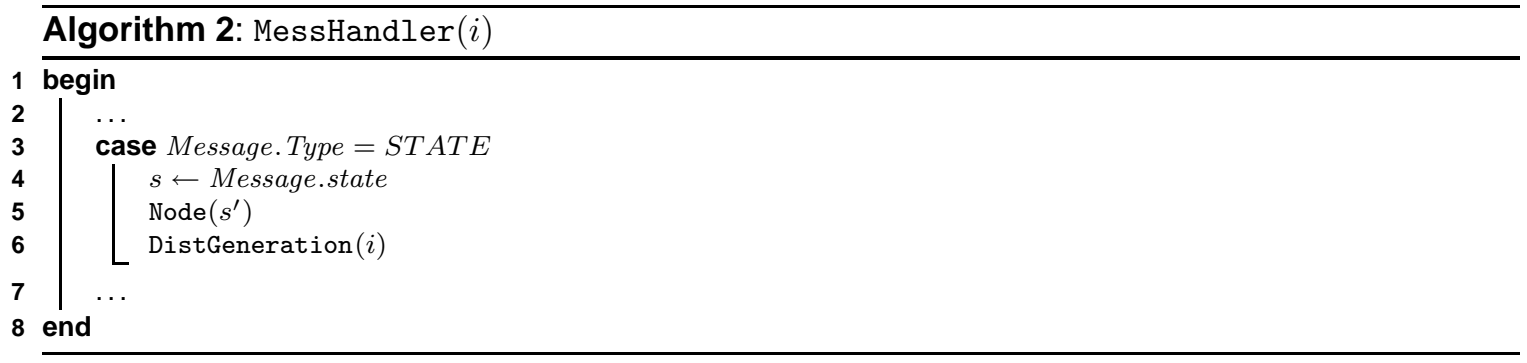

The main procedure contains a waiting loop which stops when a TERMINATE message is received from the coordinator process. The DistGeneration $(i)$ procedure is invoqued when the process receives the first marking. Messages vehicle states or other information ensuring the termination of the distributed algorithm. They are all taken care of by the same procedure MessHandler(). The termination occurs when all processes have finished handling their states, and no message is in transit.

\section{DISTRIBUTED MODEL CHECKING OF CTL FORMULAS}

In this section, we present algorithms to verify CTL formulas on a distributed state space. We consider the same architecture based on coordinator and worker processes. We suppose that the state space is entirely partitioned over all the stations of the cluster, each station (process) $i$ owns the set of states $S_{i}=\{s \mid h(s)=i\}$, and each process constructed the parse tree of the CTL formula [CES86].

Each process computes the set of local states $\operatorname{Sat}_{i}(\varphi)$ which satisfy $\varphi$, such that:

$$
\operatorname{Sat}(\varphi)=\bigcup_{i=0, N-1} \operatorname{Sat}_{i}(\varphi) .
$$


Propositional subformulas, atomic propositions, negation and conjunction can be verified locally by each process. However, when the CTL formula includes the temporal operators $E X, A X, E U$ or $A U$, communications between processes to check the non-local neighbouring states become necessary. We consider only the operators $E X, E U$ and $A U$ which cover all the CTL formulae. The operator $E G$ could be considered instead of $A U$, but its verification requires the computation of the stongly connected components in a distributed setting.

To compute $\operatorname{Sat}_{i}(\varphi)$ for a formula $\varphi$ containing subformulas, we proceed inductively by labelling with $\varphi$ the states satisfying $\varphi$. We assume that the sets of local states satisfying these subformulas are already computed (labelled). This is always the case when the parse tree is used.

\subsection{Propositional CTL formulae}

When $\varphi$ is a propositional CTL formula, all processes compute their set of local states $\operatorname{Sat}_{i}(\varphi)$ which satisfy $\varphi$, independently of each other, using algorithm 3.

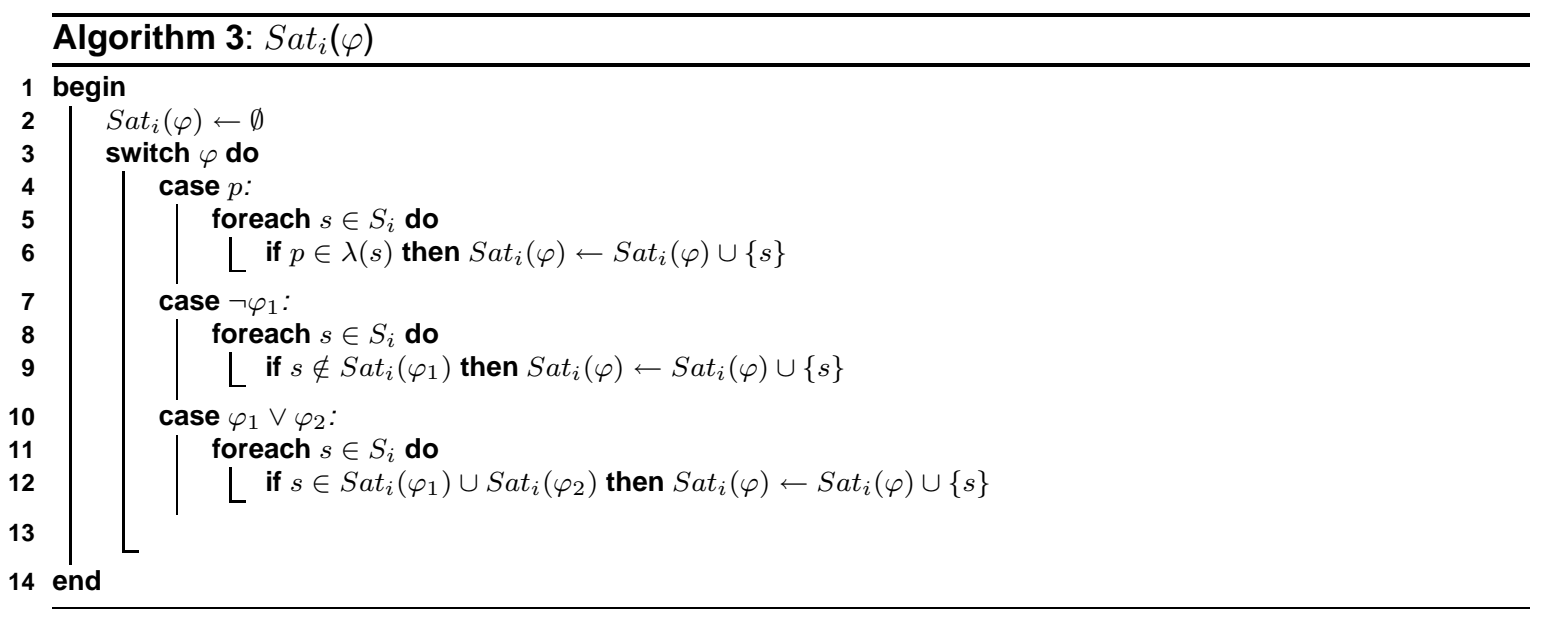

Since there is no need for communications to perform the distributed checking of logical formulas, we can expect a linear speedup, provided the hash function is appropriate.

4.2. neXt CTL formulae: $\psi=E X \varphi$ and $\psi=A X \varphi$

Assume that the local set $\operatorname{Sat}_{i}(\varphi)$ is known. To compute the set of states satisfying $\psi=E X \varphi$, each machine executes algorithm 4.

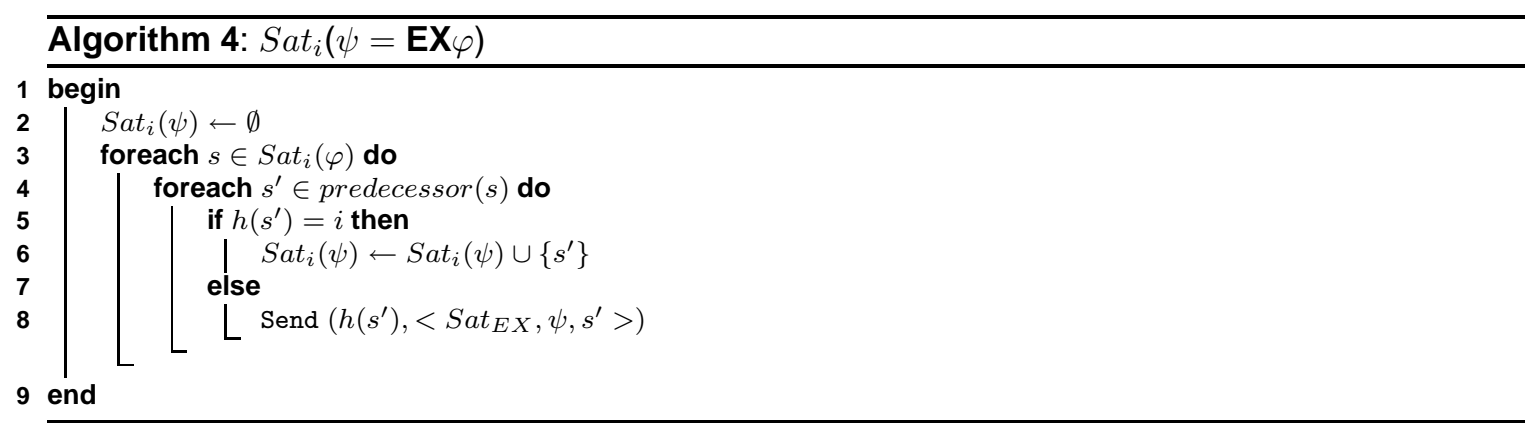

Every process $i$ adds each local predecessor $s^{\prime}$ of the states which satisfy $\varphi$ to $\operatorname{Sat}_{i}(\psi)$, and sends messages to the processes owning distant predecessors to do so. When a process $j$ receives a state $s$, it adds it to $\operatorname{Sat}_{j}(\psi)$ (see algorithm 7, lines 3-4). There is no need for process $j$ to reply to process $i$. 
Since we proceed in a backward manner, it is not necessary to wait for sets $\operatorname{Sat}_{j}(\varphi)$ to be known by the other processes $j \neq i$. Thus, the processes can work independently of each other and in a parallel way. Hence, we can also expect a linear speedup.

The satisfaction of $A X \varphi$ can be obtained by checking $\neg E X \neg \varphi$.

\subsection{Exist Until formulae: $\psi=\mathbf{E}\left(\varphi_{1} \cup \varphi_{2}\right)$}

To evaluate the CTL formula $\psi=E\left(\varphi_{1} \cup \varphi_{2}\right)$, we assume that $\operatorname{Sat}_{i}\left(\varphi_{1}\right)$ and $\operatorname{Sat}_{i}\left(\varphi_{2}\right)$ are known by each station $i$. This means that processes must wait for each other until these sets are known in all processes. Then, to compute the set $\operatorname{Sat}_{i}(\psi)$, each process executes algorithm 5 .

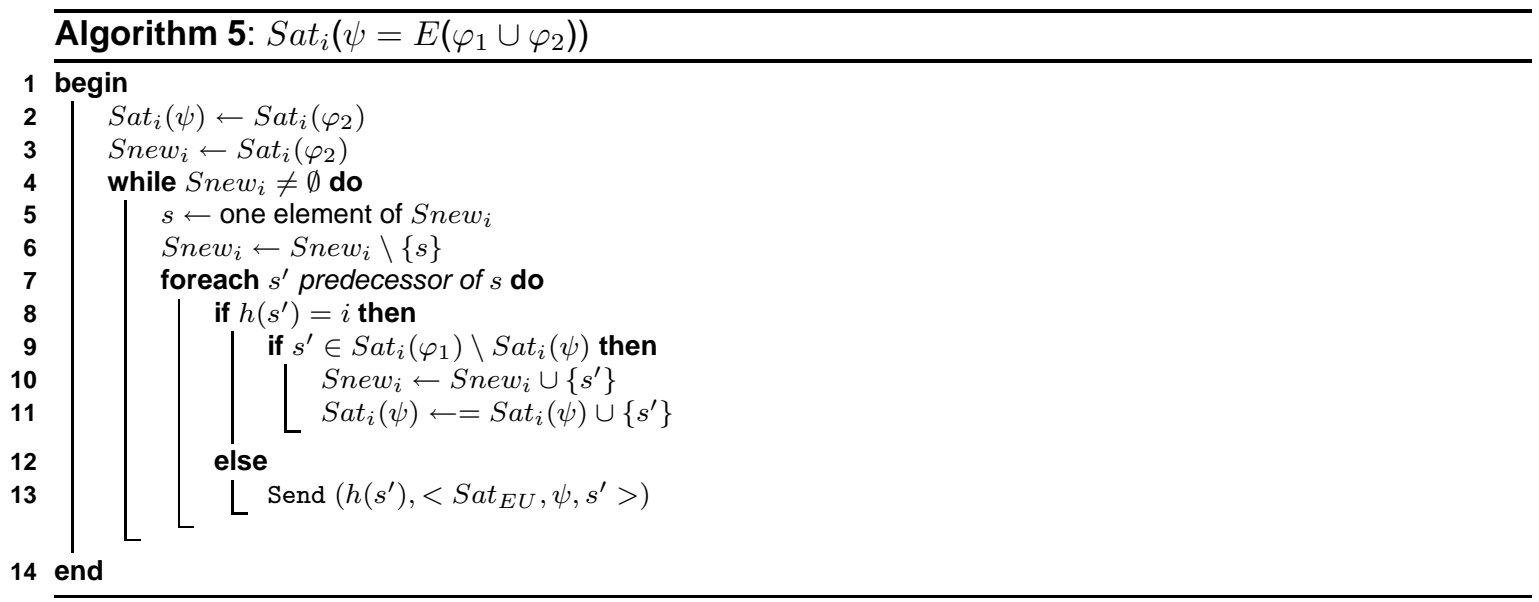

A set $S n e w_{i}$ is used to contain the new states which satisfy $\psi$. Initially, $\operatorname{Snew}_{i}$ contains $\operatorname{Sat}_{i}\left(\varphi_{2}\right)$. At each iteration a state $s$ is removed from $S n e w_{i}$, and every predecessor $s^{\prime} \notin \operatorname{Sat}_{i}(\psi)$ satisfying $\varphi_{1}$, then satisfies $\psi$, is included in $S n e w_{i}$ to check whether its predecessors satisfy $\varphi_{1}$ too. For distant predecessors a message is sent to their owner process. When a process $j$ receives a such message it adds the state to $S_{n e w}$ and to $\operatorname{Sat}_{i}(\psi)$ (see algorithm 7, lines 10-14).

\subsection{For All Until CTL formulae: $\psi=A\left(\varphi_{1} \cup \varphi_{2}\right)$}

Similar ideas are used to compute the set of states which verify the AU formulae. $\operatorname{Sat}_{i}\left(\varphi_{1}\right)$ and $\operatorname{Sat}_{i}\left(\varphi_{2}\right)$ being known for all processes, $\operatorname{Sat}_{i}(\psi)$ is computed for each process $i$.

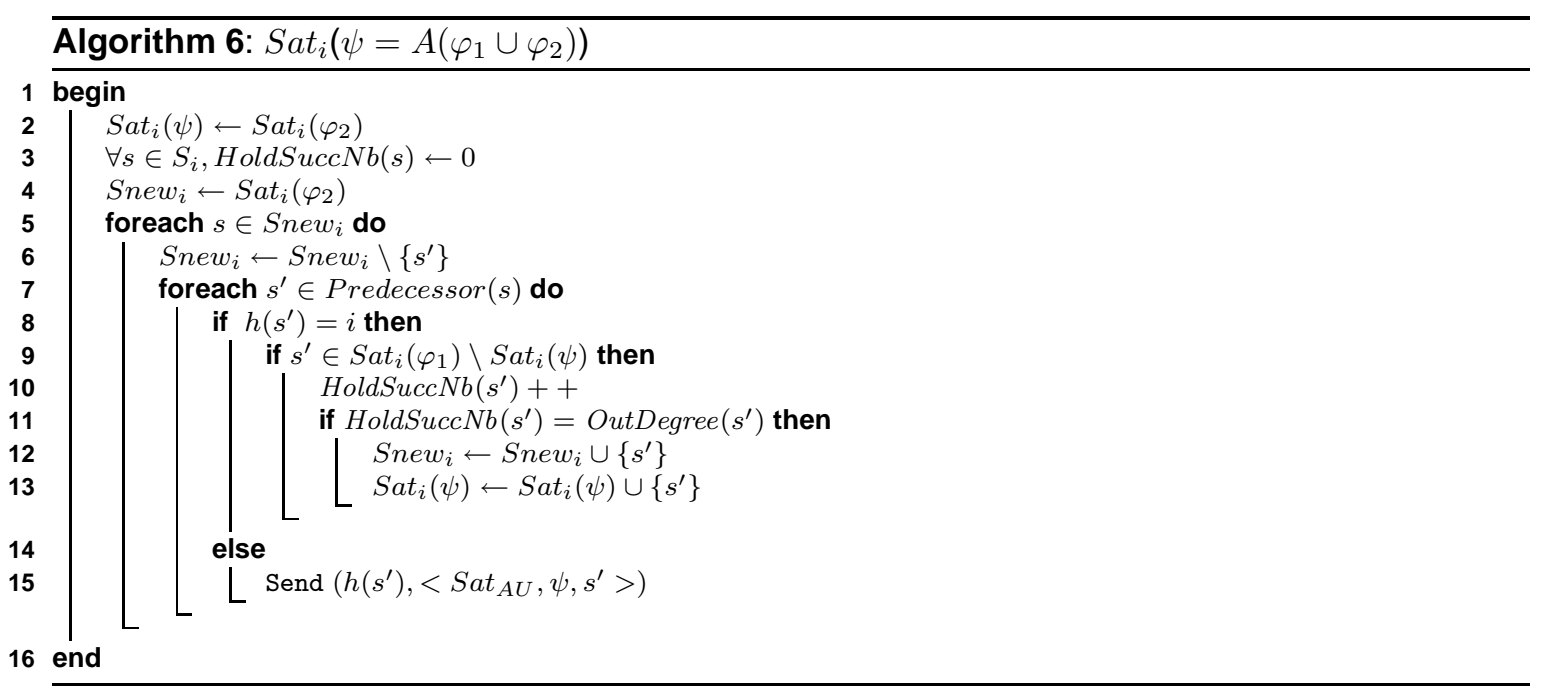




\subsection{Specific messages}

Communications are required when successors and predecessors are not handled by the same process. Hence, function MessHandler() is extended so as to handle the messages concerning the formulae evaluation as described in algorithm 7.

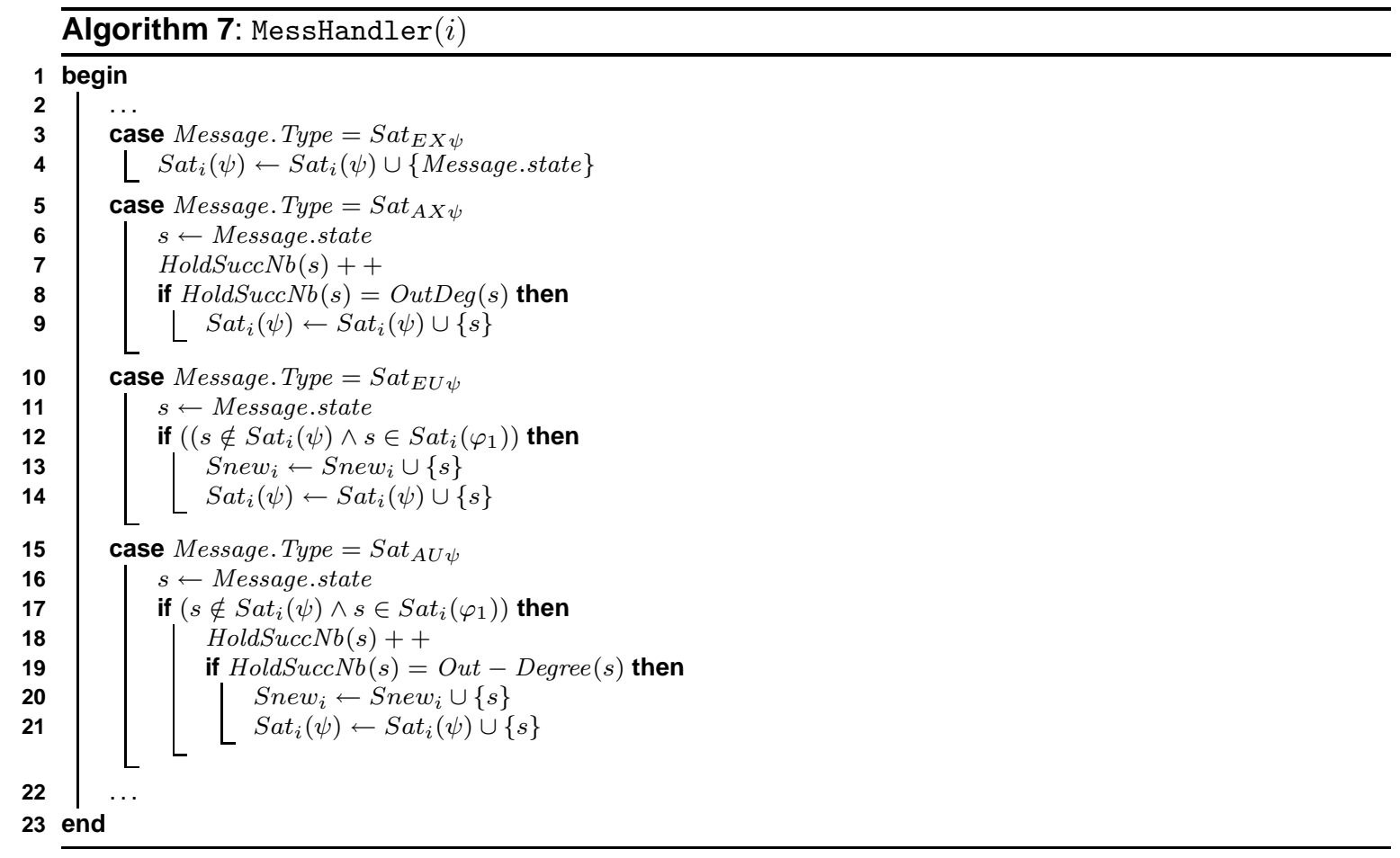

The termination of the verification of a formula $\psi$ occurs when the initial state is added to $\operatorname{Sat}_{i}(\psi)$ or when all processes end the labelling of their own states. In the last case, the formula is not satisfied, and we propose a distributed search of the counterexample.

\section{DISTRIBUTED COUNTEREXAMPLE SEARCH}

The model-checking algorithm either terminates with success, if the property holds, or with failure otherwise. In this latter case, a counterexample provides the designer with the reasons for the failure and can be helpful to find subtle errors in the system. A large class of temporal logics admit counterexamples as simple tree-like structures [CJLV02]. It consists in a structure which shows that the negation of the formula is true.

Here, we propose to determine a counterexample from the distributed state space, allowing the use of explicit state enumeration techniques on very large models, by exploiting resources of multiple stations of a cluster. In this section, we focus on determining the counterexample for a formula using the temporal operators $A U$ and $E U$ since they are the most challenging cases.

The counterexample search is done in a depth first manner, starting from the initial state $s_{0}$. It is easy to determine the counterexample in the case of a formula $\psi=A X \varphi$ : when it does not hold, there exists a successor $s$ of $s_{0}$ such that $s \not \forall \varphi$. Thus, the counterexample is the sub-graph containing only $s_{0}$ and its successors not satisfying $\varphi$.

However, for both formulae $A\left(\varphi_{1} \cup \varphi_{2}\right)$ and $E\left(\varphi_{1} \cup \varphi_{2}\right)$, the corresponding counterexample consists in all the paths $\pi=s_{0} s_{1} \ldots$ such that:

$$
\exists i\left(i \geq 0 \wedge s_{i} \models\left(\neg \varphi_{1} \wedge \neg \varphi_{2}\right) \wedge \underset{\text { or }}{\left.\forall j\left(0 \leq j<i \Rightarrow s_{j} \models\left(\varphi_{1} \wedge \neg \varphi_{2}\right)\right)\right)}\right.
$$




$$
\exists i\left(i \geq 0 \wedge \exists j\left(j>0 \wedge s_{i}=s_{i+j} \wedge \forall k\left(0 \leq k<i+j \Rightarrow s_{k} \models\left(\varphi_{1} \wedge \neg \varphi_{2}\right)\right)\right)\right.
$$

The first condition means that $\pi$ contains a succession of states $s_{0}, s_{1}, \ldots s_{i-1}$ where $\varphi_{1}$ holds, and in $s_{i}$ neither $\varphi_{1}$ nor $\varphi_{2}$ holds. In the second condition, the path $\pi$ contains a cycle where $\varphi_{1}$ holds but not $\varphi_{2}$. It is then possible to execute the cycle infinitely often, thus never reaching a state where $\varphi_{2}$ holds.

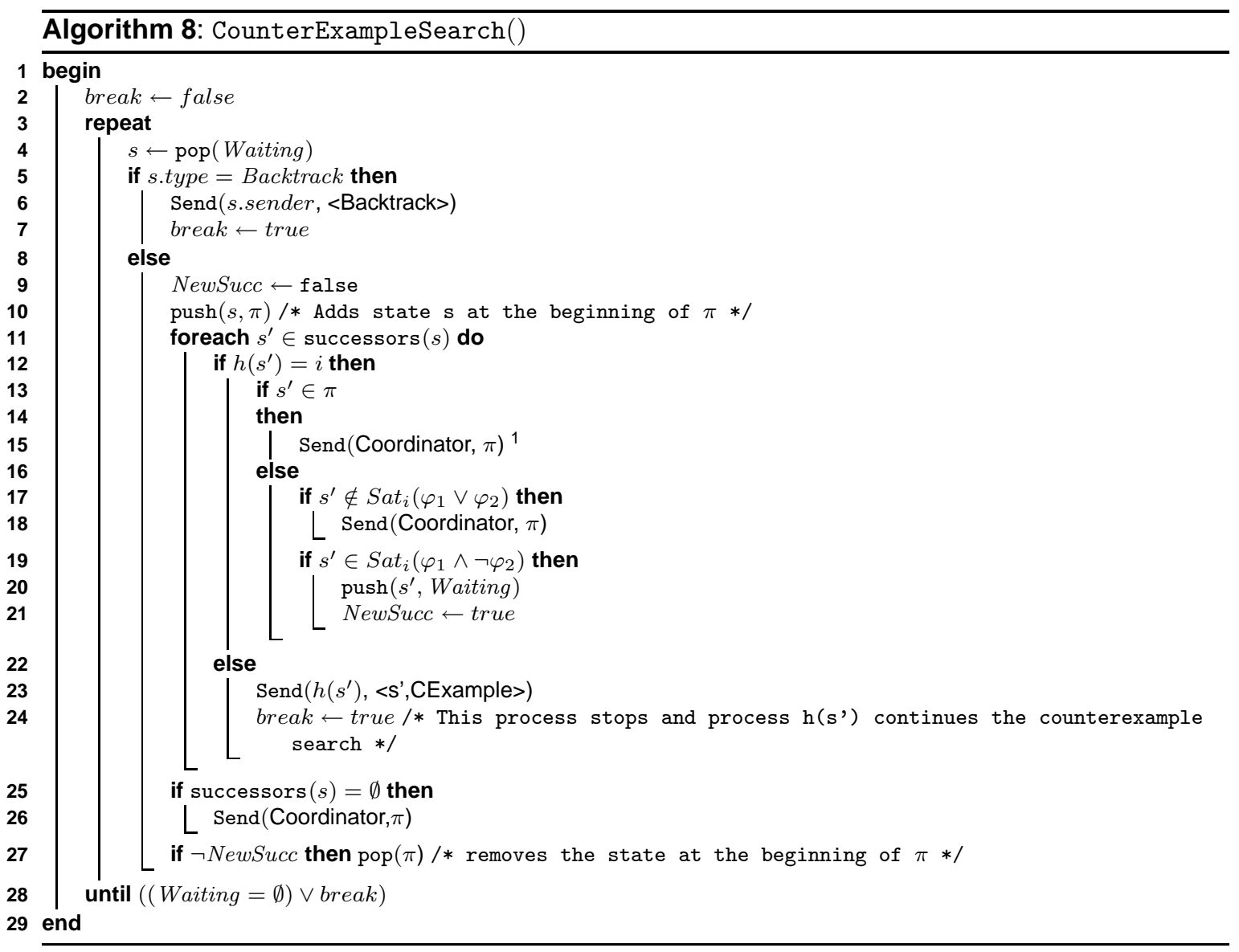

To exhibit the counterexample (algorithm 8), each process uses stack Waiting. Initially, it contains the initial state $s_{0}$ in the process $h\left(s_{0}\right)$.

$\pi$ will contain the local part of the path corresponding to a counterexample. The elements of $\pi$ are labelled using a global counter allowing the coordinator to reorder the entire counterexample.

The Waiting stack can contain either states or backtrack tags. The states are those in which $\varphi_{1}$ holds but not $\varphi_{2}$. The backtrack tags are used when the backtrack leads to a distant predecessor.

At each iteration the state $s$ at the top of stack Waiting is extracted and added to path $\pi$. Each local successor $s^{\prime}$ of $s$ can be in one of the following cases:

- $s^{\prime}$ satisfies $\varphi_{1}$ but not $\varphi_{2}$. Then $s^{\prime}$ is added to the stack Waiting extending the path which could be a counterexample.

- $s^{\prime}$ belongs to $\pi$. This means that $\pi$ contains a cycle whose states satisfy $\varphi_{1}$ but not $\varphi_{2}$. Then, path $\pi$ is a counterexample for formula $\psi$.

- $s^{\prime}$ does not satisfy $\varphi_{1}$ nor $\varphi_{2}$. Then $\pi$ constitutes a counterexample.

\footnotetext{
${ }^{1}$ Sends the part of the path or cycle constituting the counterexample to the coordinator which reorders the states to display the entire counterexample.
} 
For the distant successors, messages are sent to their owners so as to be handled. If $s$ has no successor, or no successor satisfying either $\varphi_{1}$ or $\varphi_{2}$, then $\pi$ constitutes also a counterexample (lines 17 and 25).

When the top of the stack contains a backtrack tag, the next state to pop is distant. A message is then sent to its owner to pursue processing.

The process $h\left(s_{0}\right)$ starts the counterexample search as described in algorithm 9 when it receives a CounterExample message.

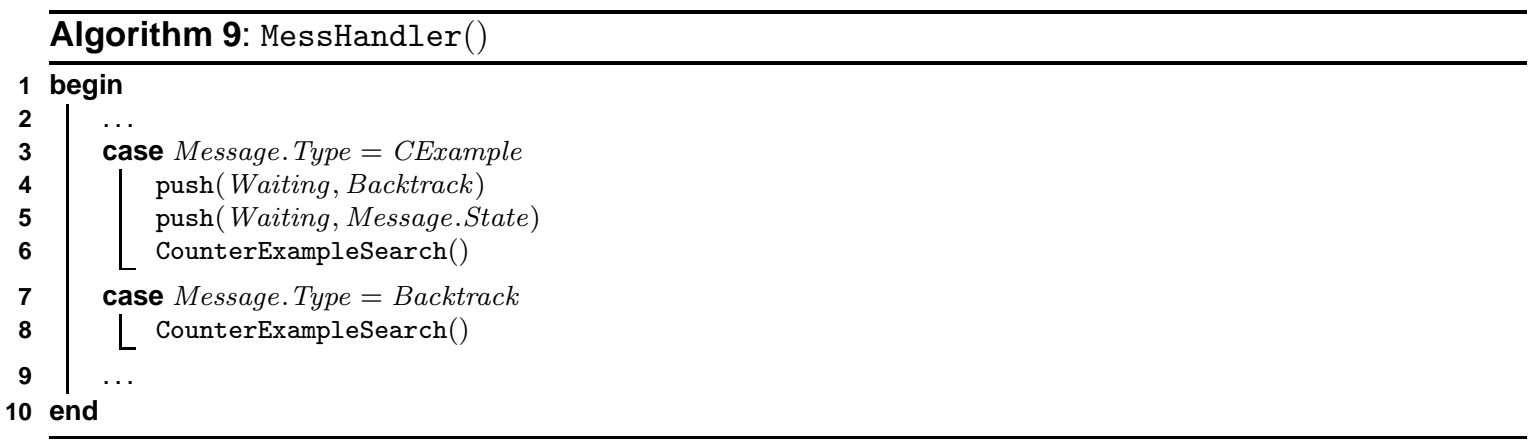

Cycle detection can be performed using depth-first exploration. However, if the exploration is carried out in a parallel way, some cycles might be overlooked. Hence, when a process sends a state, it remains idle until it receives a Backtrack message or another state to be visited.

\section{IMPLEMENTATION AND EXPERIMENTAL RESULTS}

The previous algorithms were implemented within a prototype tool. Given a Petri net, the reachability graph is generated on a cluster of stations, and then a CTL formula is verified. In this section, we give the results obtained for the dining philosophers problems. Other problems like the distributed database managers were also studied. Whenever the formula is not satisfied, the subgraph corresponding to the counterexample is entirely computed, and reported to the user. The tests were carried on a cluster composed of 12 stations (Pentium IV with 512 Mbytes of memory).

\subsection{The dining philosophers}

The dining philosophers problem, introduced by Dijkstra, concerns the problem of resources allocation between processes. Several philosophers sit around a circular table. There is a fork between each pair of neighbouring philosophers. Each philosopher spends his life alternating thinking and eating phases, and may arbitrarily decide to use either the fork to his left or the one to his right, but needs both of them to eat.

The states of the dining philosophers problem can be partitioned into sets $S_{1}, S_{2} \ldots$ such that $S_{i}$ does not contain all the states corresponding to $i$ eating philosophers. Indeed, these states are not linked by any transition, they constitute a stable, so it is more interesting to store them on different stations. On the contrary, a state where there are $i$ eating philosophers is only linked to states where there are $i-1$ and $i+1$ eating philosophers, such that there are respectively $i-1$ and $i$ eating philosophers common to both states. Hence, our hash function tries to store them on the same station.

The experimental results for this problem are given in table 1 . We can note that the number of cross arcs compared to the total number of transitions, given by the ratio $N_{1} / N_{2}$, grows to reach more then 8 , which means that there is one traversal transition for more then eight local transitions. The ratio $N_{3} / N_{2}$ shows that the number of messages is approximately 2 messages for each traversal transition. A superlinear speedup is obtained, as shown in the last two columns. 


\begin{tabular}{|c|r|r|r|r|r|r|r|r|}
\hline \begin{tabular}{c|}
$\mathrm{Nb}$ \\
Phil
\end{tabular} & $\begin{array}{r}\mathrm{Nb} \\
\text { States }\end{array}$ & $\begin{array}{r}\text { Nb Trans } \\
\left(N_{1}\right)\end{array}$ & $\begin{array}{r}\text { Nb Cross. } \\
\operatorname{arcs}\left(N_{2}\right)\end{array}$ & $N_{1} / N_{2}$ & $\begin{array}{r}\text { Nb Mess. } \\
\left(N_{3}\right)\end{array}$ & $N_{3} / N_{2}$ & $\begin{array}{r}\text { Time } \\
\text { G. }(\mathrm{sec})\end{array}$ & $\begin{array}{r}1 \text { proc } \\
\text { Time }\end{array}$ \\
\hline 5 & 11 & 30 & 18 & 1.67 & 36 & 2.00 & $<0.01$ & $<0.01$ \\
10 & 123 & 680 & 242 & 2.81 & 494 & 2.03 & $<0.01$ & 0.01 \\
15 & 1,364 & 11,310 & 2,731 & 4.14 & 5477 & 2.01 & 0.06 & 0.38 \\
20 & 15,127 & 167,240 & 30,236 & 5.53 & 60,493 & 2.00 & 2.52 & 13.48 \\
25 & 167,761 & $2,318,400$ & 315,718 & 7.34 & 631,587 & 2.00 & 32.58 & 216.09 \\
30 & $1,728,813$ & $28,686,031$ & $3,572,821$ & 8.03 & $7,156,116$ & 2.00 & 7547.45 & $\gg^{2}$ \\
\hline
\end{tabular}

TABLE 1: Distributed generation

We analysed several formulae which do not hold, e.g. two thinking philosophers can start eating at the same time,

$$
E[(\operatorname{thinking}(i) \wedge \operatorname{thinking}(j)) \cup(\operatorname{eating}(i) \wedge \operatorname{eating}(j))]
$$

or two neighbors eating at same time,

$$
E F(\operatorname{eating}(i) \wedge \operatorname{eating}(i+1 \bmod \mathbf{n}))
$$

The analysis of these formulae always leads to a failure, and a counterexample search. The second formula gives only cycles involving the entire state space.

The results obtained for the first formula are given in tables 1 and 2 where the last column indicates the computation time with a single process (i.e. not distributed).

\begin{tabular}{|c|c|c||r|r|r|r|c|}
\hline $\begin{array}{c}\text { Nb } \\
\text { Phil }\end{array}$ & $\begin{array}{c}\text { Verif. } \\
\text { Time (sec) }\end{array}$ & $\begin{array}{c}1 \text { proc } \\
\text { Verif Time }\end{array}$ & $\begin{array}{r}\text { Tot. Nb } \\
\text { of CE }\end{array}$ & $\begin{array}{r}\text { Paths } \\
\text { CE }\end{array}$ & $\begin{array}{c}\text { Mess. Nb } \\
1 \text { st CE }\end{array}$ & $\begin{array}{r}\text { Tot. Nb } \\
\text { Mess }\end{array}$ & $\begin{array}{c}\text { Max CPU } \\
\text { time }\end{array}$ \\
\hline 5 & $<0.01$ & $<0.01$ & 11 & 4 & 17 & 49 & $<0.01$ \\
10 & $<0.01$ & 0.01 & 218 & 42 & 24 & 480 & 0.01 \\
15 & 0.03 & 0.18 & 3,502 & 267 & 31 & 5,265 & 0.12 \\
20 & 0.18 & 0.77 & 49,917 & 5,168 & 49 & 52,221 & 3.46 \\
25 & 0.84 & 4.33 & 868,345 & 95,375 & 68 & 512,614 & 39.52 \\
30 & 5.15 & $\gg^{2}$ & $10.043,424$ & 190,951 & 182 & $5,204,315$ & 149.96 \\
\hline
\end{tabular}

TABLE 2: Distributed verification and counterexample search

In table 2 the first columns give results concerning formulas verification containing the EU operator, respectively the maximum CPU time for each process and the CPU time of a single process verification. Here also we have an interesting speedup.

The second part of columns concerns the distributed counterexample search. The first column gives the total number of counterexamples, the second column the number of counterexamples which are paths (others are cycles), followed by the number of messages needed for the distributed search of the first counterexample and the total number of messages to find all the counterexamples. In the last column the maximum CPU time for each process to find all counterexamples.

\section{CONCLUSION}

In this paper, we proposed algorithms for CTL distributed model-checking. First, the state space is both generated and partitioned over a cluster of stations; this step is achieved by a set of machines which collaborate to explore the entire state space. Then, the same set of machines is used to achieve a distributed model-checking of CTL formulas. We have adapted the sequential algorithms used to verify the CTL formulas to the distributed case. This permits to model check systems using explicit representation of the states, taking advantage of the memory of all the involved machines. A counterexample, based on a partitioned search, is given whenever the CTL formula is not

\footnotetext{
${ }^{2} \gg$ in the table means that more too much memory was required for the example to be processed on a single machine.
} 
satisfied. Several tests were performed over a cluster of workstations, a super-linear speedup is obtained for large problems, also allowing for verification of examples not amenable on a single machine. Future work will include large case studies issued from industrial size applications.

\section{REFERENCES}

[[BBC03a]] J. Barnat, L. Brim, and J. Chaloupka. Distributed memory LTL model checking based on breadth first search. Technical report, Mazarky University Brno, October 2003.

[[BBC03b]] J. Barnat, L. Brim, and J. Chaloupka. Parallel breadth-first search LTL modelchecking. In Proc. of the $18^{\text {th }}$ IEEE International Conference on Automated Software Engineering (ASE'03), pages 106-115. IEEE Computer Society, 2003.

$\left[\left[\mathrm{BBF}^{+}\right.\right.$01]] B. Bérard, M. Bidoit, A. Finkel, F. Laroussinie, A. Petit, L. Petrucci, and Ph. Schnoebelen. Systems and Software Verification: model-checking techniques and tools. Springer, 2001. ISBN 3-540-41523-8.

[[BBW02]] M. Leucker B. Bolling and M. Weber. Local parallel model checking for the alternationfree $\mu$-calculus. In proceeding of the 9th International SPIN Workshop on Model checking of Software (SPIN'02), 2318, 2002.

[[BCM ${ }^{+}$92]] J.R. Burch, E.M. Clarke, K.L. McMillan, D.L. Dill, and L.J. Hwang. Symbolic modelchecking : $10^{20}$ states and beyond. Information and Computation, 98(2):142-170, 1992.

[[BCY02] ] Lubos Brim, Jitka Crhova, and Karen Yorav. Using assumptions to distribute ctl model checking. ENTCS, Vol 68, Issue 04, ELSEVIER, 2002.

[[BH05] ] Alexander Bell and Boudewijn R. Haverkort. Sequential and distributed model checking of petri nets. Int. J. Softw. Tools Technol. Transf., 7(1):43-60, 2005.

[[Bou05] ] M. Bourahla. Distributed CTL model checking. Software, IEE Proceedings, 152(6):297-308, 2005.

[[BP07] ] C. Boukala and L. Petrucci. Towards distributed verification of Petri nets properties. In Proc. 1st International Workshop on Verification and Evaluation of Computer and Communication Systems (VECOS'07), Algiers, Algeria, eWiC, pages 15-26. British Computer Society, May 2007.

[[Bry92] ] R.E. Bryant. Symbolic boolean manipulation with ordered binary decision diagrams. ACM Computing surveys, 24(3):293-318, 1992.

[[BvH03] ] L. Brim, I. Černá, and L. Hejtmánek. Parallel algorithms for detection of negative cycles. Technical report, Mazarky University Brno, 2003.

[[BvMv04]] L. Brim, I. Černá, P. Moravec, and J. Šimša. Accepting predecessors are better than back edges in distributed LTL model checking. In Proc. FMCAD'04, volume 3312 of Lecture Notes in Computer Science, pages 352-366. Springer, November 2004.

[[CCBF94]] S. Caselli, G. Conte, F. Bonardi, and M. Fontanesi. Experiences on SIMD massively parallel GSPN analysis. volume 794 of Lecture Notes in Computer Science, pages 266-283, 1994.

[[CES86] ] E. M. Clarke, E. A. Emerson, and A. P. Sistla. Automatic verification of finitestate concurrent systems using temporal logic specifications. ACM Transactions on Programming Languages and Systems, 8(2):244-263, April 1986.

[[CGN98]] G. Ciardo, J. Gluckman, and D. Nicol. Distributed state space generation of discretestate stochastic models. INFORMS Journal on Computing, 1998.

[[CJLV02]] E. M. Clarke, S. Jha, Y. Lu, and H. Veith. Tree-like counterexamples in model checking. In Proc. IEEE Symposium on Logic in Computer Science (LICS'02). IEEE Computer Society Press, July 2002.

[[GMS01]] H. Garavel, R. Mateescu, and I. Smarandache. Parallel state space construction for model-checking. In Proc. SPIN workshop, volume 2057 of Lecture Notes in Computer Science, pages 217-234. Springer, 2001.

[[Hol03] ] G. Holzmann. The SPIN model-checker. Addison-Wesley, 2003.

[[IB06] ] C. P. Inggs and H. Barringer. CTL* model checking on a shared-memory architecture. Formal Methods in System Design, 29(2):135-155, 2006.

[[KM04] ] R. Kumar and E. G. Mercer. Load balancing parallel explicit state model checking. In Proc. of PDMC, volume 128 of Electronic Notes in Theoretical Compter Science, 2004. 
[[KP04] ] L. Kristensen and L. Petrucci. An approach to distributed state exploration for coloured Petri nets. In Proc. 25th Int. Application and Theory of Petri Nets (ICATPN'2004), volume 3099 of Lecture Notes in Computer Science, pages 474-483. Springer, 2004.

[[LS99] ] F. Lerda and R. Sisto. Distributed-memory model checking in SPIN. In Proc. of the SPIN workshop, volume 1680 of Lecture Notes in Computer Science. Springer, 1999.

[[McM98]] K. McMillan. An introduction to model-checking. In Proc. of the summer school MOVEP'98, Nantes, July 1998. 